\title{
Solvent resistant nanofiltration for product purification and catalyst recovery in click chemistry reactions
}

\author{
A. Cano-Odena, ${ }^{[a]}$ P. Vandezande, ${ }^{[a],[1]}$ D. Fournier, ${ }^{[b],[2]}$ W. Van Camp, ${ }^{[b]}$ F. E. Du Prez, ${ }^{[b]}$ I. \\ F.J. Vankelecom ${ }^{[a],[*]}$
}

\begin{abstract}
The quickly developing field of 'click' chemistry would undoubtedly benefit from the availability of an easy and efficient technology for product purification, reducing the potential health risks associated with the presence of copper in the final product. Therefore, solvent resistant nanofiltration (SRNF) membranes were developed that could selectively
\end{abstract}

separate 'clicked' polymers from the copper catalyst and solvent. By operating these solvent-stable crosslinked polyimide membranes in diafiltration mode, up to $98 \%$ of the initially present copper could be removed through the membrane together with the DMF solvent, while the polymer product was almost completely retained. This paper also presents the first SRNF-application in which a catalyst is permeated and the reaction product retained.

\section{Introduction}

The need for developing new and versatile materials with improved properties has recently led to the search for new synthetic routes. The so-called 'click' chemistry receives particular attention as it provides an easy and elegant synthetic tool to couple chemical compounds. Among the different 'click' reactions described by Sharpless et al., ${ }^{[1]}$ copper (I) catalyzed Huisgen 1,3-dipolar cycloadditions between azides and terminal alkynes are nowadays one of the most used (Figure 1). The advantages of such reactions are numerous, i.e. a high selectivity and functional group tolerance, mild reaction conditions and quantitative yields. Furthermore, the possibility of tailoring the coupling molecules by easily introducing alkyne and azide functions at the required location, has led to the

[a] Dr. A. Cano-Odena, Ir. P. Vandezande, Prof. I. F. J. Vankelecom Centre for Surface Chemistry and Catalysis

Faculty of Bioscience Engineering, Katholieke Universiteit Leuven Kasteelpark Arenberg 23 - box 2461, B-3001 Leuven, Belgium Fax: (+32) 16-321998

E-mail: ivo.vankelecom@biw.kuleuven.be

[b] Dr. D. Fournier, Dr. W. Van Camp, Prof. F. E. Du Prez, Polymer Chemistry Research Group, Department of Organic Chemistry, Ghent University

Krijgslaan 281 S4, B-9000 Gent, Belgium

[1] Present address: Flemish Institute for Technological Research (VITO), Boeretang 200, B-2400 Mol, Belgium

[2] Present address: Laboratoire de Chimie Organique et Macromoléculaire (UMR 8009), Université des Sciences et Technologies de Lille 59655 Villeneuve d'Ascq Cedex rapid development of 'click' chemistry in many fields of application. ${ }^{[2-5]}$ Especially in polymer and materials science, postmodifications using 'click' reactions represent an efficient and versatile approach for end-functionalization of polymers, synthesis of block copolymers, hyperbranched and dendritic macromolecules and star-shaped polymers. ${ }^{[4-7]}$

Figure 1. Principle of copper (I)-catalyzed Huisgen 1,3-dipolar cycloaddition.

Due to the potentially toxic contamination of 'click' products, effective removal of ligand-stabilized copper (I) catalysts from postreaction solutions is of great importance to meet the specification limits for medicinal use, where significant levels of heavy metal residues are understandably unacceptable. ${ }^{[8]}$ Active pharmaceutical ingredients are mostly synthesized in reactions that utilize homogeneous catalysts, which frequently present major downstream separation challenges. Even though copper is considered as an essential element with a clear nutritional value, guidelines of the European Medicines Agency have set the permitted daily exposure (PDE) of patients to copper at $50 \mu \mathrm{g} \cdot \mathrm{kg}^{-1}$ for oral doses and 10 $\mu \mathrm{g} . \mathrm{kg}^{-1}$ for parenterally administered drugs. ${ }^{[9]}$

Recently, great interest was attributed to the development of metalfree strategies for the click reaction of azides and alkynes. Bertozzi and coworkers ${ }^{[10]}$ synthesized strained cycloalkynes to enhance the 
reactivity of the alkynes, a strategy that was further improved by adding electron-withdrawing substituents on the $\alpha$-position of the triple bond. Another approach makes use of substituted dibenzocyclooctyenes. ${ }^{[11]}$ Lutz recently summarized the efforts towards metal-free azide-alkyne cycloadditions, and concluded that the developed strategies will not replace the copper (I) catalyzed reactions in the near future. ${ }^{[12]}$ Still in search for avoiding the use of a copper catalyst, a number of alternative 'click' strategies have been recently reported. ${ }^{[13]}$ Among these, Diels-Alder 'click' reactions $^{[14]}$, thiol-based 'click' reactions ${ }^{[15]}$ and in particular hetero Diels-Alder cycloaddition reactions ${ }^{[16]}$ are of great interest. However, the copper (I) catalyzed azide-alkyne cycloaddition is expected to remain by far the most widely applied 'click' reaction, thanks to the simple and readily available building blocks. ${ }^{[13]}$

Post-reaction purification technologies commonly used in the separation of homogeneous 'click' chemistry catalysts include distillation, ${ }^{[17]}$ extraction, ion-exchange resins, ${ }^{[18]}$ adsorption, ${ }^{[19]}$ ionic liquids, ${ }^{[20,21]}$ or supercritical fluids. ${ }^{[22]}$ These work-up methods are usually destructive and require an additional step to gain back the catalyst prior to its re-use, which often leads to partial catalyst loss and thus additional costs. At present, hardly any industrially used separation technique is aimed at the recovery of homogeneous catalysts in their active form, but rather at obtaining a pure product whilst recovering the metal in a form that may be recycled to a catalyst manufacturer.

Alternatively, the 'click' catalyst can be heterogenized by covalent or non-covalent immobilization on e.g. silica beads, polystyrene or mesoporous supports. ${ }^{[23-25]}$ Another approach is the immobilization of the catalyst onto soluble supports ${ }^{[26,27]}$ that can be retained by ultra- or nanofiltration (NF) membranes. However, the use of heterogeneous catalysts results, in general, in slower reaction rates because the ligands do not match the optimal steric and electronic requirements achieved in homogeneous click reactions. ${ }^{[8,19]}$

With the recent development of polymeric membranes, solvent resistant nanofiltration (SRNF) ${ }^{[28]}$ appears to be a promising alternative permitting the use of homogeneous 'click' catalysts and solving the problems arising from the catalyst's presence in the product after reaction. Such approach would not only allow to remove copper from the post-reaction mixture, but also to recover the catalyst for re-use in subsequent reaction runs. The chemical stability and long-term performance of the membranes are crucial, since 'click' reactions are often carried out in polar aprotic solvents such as dimethylformamide (DMF). These solvents are particularly challenging for polymeric membranes since they are typically used to dissolve polymers prior to membrane formation via phaseinversion.

Important efforts have been made over the past years to separate 'off-the-shelf' homogeneous catalysts from their reaction mixtures with SRNF membranes in order to recycle the catalyst and/or facilitate product purification. SRNF-coupled recovery of transition metal complexes has been amply reported in literature ${ }^{[17,29-36]}$, such as for Pd coupling catalysts, the Co Jacobsen catalyst, Ru metathesis catalysts, and Rh hydroformylation catalysts. A hybrid process based on the combined use of a commercial polyimide (PI) membrane and adsorbents has been proposed for Pd removal from post-coupling reaction products. ${ }^{[37]}$ The use of a commercial ceramic NF membrane has been recently reported to recover 'click'synthesized dendritic phosphine ligands attached to a soluble support, which were applied in the Pd-catalyzed coupling of aryl halides and phenylboronic acid. ${ }^{[38]}$ However, the use of SRNF membranes to purify 'click' products and recycle the unmodified copper catalyst has never been reported.

In the present study, SRNF will be applied to different 'click' postreaction mixtures using DMF and THF as solvents, with the objective to purify the polymeric product and to maximally recover the catalyst. The recently developed crosslinked PI membranes ${ }^{[39,40]}$ are excellent candidates, since they are stable in polar aprotic solvents and allow easy tuning of their molecular weight cut-off (MWCO) by modifying the synthesis parameters. Several membranes will be prepared and screened for their separation properties in the filtration of a typical 'click' post-reaction mixture. An optimal membrane will then be selected and applied in a diafiltration experiment in which the impurities - copper and any residual reagent - are progressively 'washed out' together with the solvent. In contrast to earlier SRNF/catalysis studies where the catalyst has always been retained by the membrane, ${ }^{[28]}$ the present work envisages retention of the relatively large polymeric product and permeation of the catalyst. Such approach entails important advantages since the purified product is also concentrated at the feed side of the membrane, hence facilitating subsequent isolation, while the catalyst remains basically in its original environment, thus minimizing de-activation processes due to increased concentration.

\section{Results and Discussion}

\section{Membrane screening}

Three asymmetric PI membranes were prepared via phase inversion ${ }^{[41]}$ and subsequently crosslinked with aromatic diamines to allow filtrations in demanding solvents such as DMF and THF. ${ }^{[39]}$ Phase inversion involves a controlled transformation of a polymeric solution into a solid film, which was in this case induced by immersion of a cast polymer film in a non-solvent bath (immersionprecipitation). Thanks to the miscibility between the solvent in the film and the water in the non-solvent bath, the latter diffuses into the polymer film and procures the solidification of the developing membrane structure. Crucial for the formation of a selective top layer, and thus for NF selectivity, is a short exposure of the cast film to the atmosphere (60 $\mathrm{s}$ in this case) prior to immersion in the coagulation bath, since this allows partial evaporation of the solvents from the film surface. ${ }^{[41]}$ PI membranes differ in polymer concentration and/or NMP:THF solvent ratio of the dopes they are cast from, as can be seen in Table 5 (see experimental section). These membranes were screened on different 'click' post-reaction mixtures obtained after reaction of typical alkyne with azide reagents and DMF as a solvent (Table 6, see experimental section), in order to tune their rejection properties. The screening was aimed at an efficient purification and a high yield of the formed 'click' polymer, i.e. a maximal rejection of the product by the membrane, combined with an as complete as possible passage of the copper (I) catalyst to the permeate, together with the DMF solvent and any residual reagent.

Membranes M1 and M2 were selected for the filtration of the mixture obtained after reaction between a relatively high molecular weight alkyne-functionalized polyurethane $(8100 \mathrm{~g} / \mathrm{mol})$ and $\mathrm{N}-2$ (azidoethyl)phthalimide $\left(\mathrm{PHT}-\mathrm{N}_{3}\right)$, using $\mathrm{CuBr} / \mathrm{PMDETA}$ as copper catalyst and DMF as solvent (Table 6, reaction 1). In first instance, catalyst permeation through the membrane was simply observed visually by the appearance of a greenish blue colour in the permeate, while the presence of the cycloaddition product in the permeate was determined by ${ }^{1} \mathrm{H}-\mathrm{NMR}$. As shown in Table 1, both membrane M1 and M2 are sufficiently selective to allow full product rejection, as no polymer was detected in the permeates. However, a clear difference in permeate colour was observed during filtration. While for membrane M2, an intensely blue permeate was obtained (Table 1, entry 2), filtration with membrane M1 resulted in a lightly blue filtrate (Table 1, entry 1), suggesting that the 'in situ' generated copper catalyst (molecular weight of $317 \mathrm{~g} / \mathrm{mol}$ ) was partially retained, together with the polyurethane product. Complexation of copper by polyurethanes has been reported, ${ }^{[42]}$ but does not explain the difference in permeate colour. The copper rejection depends on the MWCO of the membrane, i.e. the more dense membrane M1, cast from a more concentrated PI solution (Table 5), partially rejects 
the catalyst complex, while the more open membrane M2 is less selective and allows full catalyst permeation. This trend could be expected since a higher PI concentration in the precursor solution leads to a higher concentration at the polymer/non-solvent interface upon immersion. As a result, membranes with a denser surface structure and a lower porosity, and therefore a higher catalyst rejection, are obtained. ${ }^{[43,44]}$ This difference in MWCO can also be seen in the permeate fluxes where membrane M2 exhibits a much higher permeability than membrane M1 (Table 1).

Table 1. Membrane screening tests on 'click' post-reaction mixtures in DMF. Experiments performed in duplicate (average performances are shown). Filtration conditions: 10 bar, room temperature. the copper catalyst was formed in situ based on different concentrations of $\mathrm{CuBr} / \mathrm{PMDETA}$, i.e. 0.1 and 0.5 equivalents relative to the alkyne moieties. Catalyst and product rejections were quantitatively determined whileanalysis was performed by UV-Vis absorbance measurements and gravimetric analysis, respectively.

Table 2. Screening of the reaction conditions for the 'click' coupling of azido-PEO (2000 g/mol) with phenylacetylene (entries 1 and 2) and PBM (entries 3 and 4). Filtration conditions: membrane M3, 10 bar, room temperature, data are the average of two experiments.

\begin{tabular}{cccccc}
\hline Entry & Membrane $^{\mathbf{a}}$ & Reaction $^{\mathbf{b}}$ & $\begin{array}{c}\text { Permeability } \\
\left(\mathbf{l . m}^{-2} \cdot \mathbf{h}^{-1} \cdot \mathbf{b a r}^{-1} \mathbf{)}\right.\end{array}$ & $\begin{array}{c}\text { Permeate } \\
\text { colour }\end{array}$ & $\begin{array}{c}\text { Product in } \\
\text { permeate }\end{array}$ \\
\hline 1 & M1 & 1 & $1.3 \pm 0.2$ & light blue & no \\
2 & M2 & 1 & $3.2+0.5$ & blue & no \\
3 & M2 & 2 & $2.9+0.4$ & dark blue & yes \\
\hline
\end{tabular}

${ }^{\mathrm{a}}$ See Table 5 .

b See Table 6.

\begin{tabular}{|c|c|c|c|c|c|}
\hline Entry & Reaction $^{a}$ & $\begin{array}{c}\text { Equivalents } \\
\text { CuBr/PMDTA }^{\text {b }}\end{array}$ & $\begin{array}{c}\text { Permeability } \\
\left(\mathbf{l}^{-2} \cdot \mathbf{m}^{-1} \cdot \mathbf{h}^{-1} \cdot \text { bar }^{-1}\right)\end{array}$ & $\begin{array}{c}\text { Rejection } \\
\text { catalyst (\%) }\end{array}$ & $\begin{array}{c}\text { Rejection } \\
\text { Product } \\
(\%)\end{array}$ \\
\hline 1 & $3 a$ & 0.1 & $0.40 \pm 0.13$ & $52 \pm 10$ & $93 \pm 2$ \\
\hline 2 & $3 \mathrm{~b}$ & 0.5 & $0.9 \pm 0.4$ & $52 \pm 6$ & $93 \pm 3$ \\
\hline 3 & $4 a$ & 0.1 & $0.67 \pm 0.03$ & $45 \pm 7$ & $96 \pm 2$ \\
\hline
\end{tabular}

${ }^{\mathrm{a}}$ See Table 6.

${ }^{\mathrm{b}}$ Relative to the alkyne compound. See Table 6.

Membrane M2 was also tested in the separation of the reaction mixture obtained after cycloaddition of a low molecular weight endfunctionalized azido-PEO (1125 g/mol) and phenylacetylene in the presence of a $\mathrm{CuBr} / \mathrm{PMDETA}$ catalyst, again using DMF as a solvent (Table 6, reaction 2). In contrast to the filtrations carried out on the product mixture obtained after reaction $1,{ }^{1} \mathrm{H}-\mathrm{NMR}$ analysis revealed the presence of a significant amount product in the permeate (Table 1, entry 3 ), suggesting that the relatively low molecular weight 'clicked' polymer $(1250 \mathrm{~g} / \mathrm{mol})$ could permeate through the membrane. On the other hand, the permeate was coloured intensely blue, demonstrating that membrane M2 allows permeation of the catalyst. To avoid product permeation, another end-functionalized azido-PEO with a molecular weight around 2000 $\mathrm{g} / \mathrm{mol}$ (Table 6) was used in further reactions.

A similar screening under equal reaction conditions was also carried out on THF-based post-reaction mixtures. In this case, the membrane was unvariably coloured blue after filtration, indicating a strong catalyst adsorption on the PI membrane surface. Therefore, DMF was used as solvent in all further experiments.

Based on this qualitative preliminary study, membrane M2 was selected as the most promising membrane for copper permeation. In order to overcome the limited rejection of this membrane for relatively low molecular weight products (Table 1, entry 3) and to further improve its catalyst separation efficiency (Table 1, entry 2), a new membrane was prepared. This new membrane M3 was obtained by increasing the co-solvent:solvent (THF:NMP) weight ratio of the PI membrane precursor solution from 1:5 (M2) to 1:3 (Table 5). A higher concentration of volatile co-solvent is known to induce a thicker and/or denser top layer during the evaporation step prior to immersion of the cast film in the coagulation bath. ${ }^{[41,43,44]}$ Filtration experiments on fresh 'click' reaction mixtures in DMF were carried out with membrane M3 for two reactions with different copper concentrations. To validate the filtration process, the molecular weight of the end-functionalized azido-PEO (2000 g/mol) was chosen in such a way that the final 'click' product has a molecular weight well above the expected MWCO of the membrane, i.e. around $1000 \mathrm{~g} / \mathrm{mol}$, based on a rejection higher than 95\% for the dye rose Bengal (1017 $\mathrm{g} / \mathrm{mol})$. This azido-PEO was reacted with two different alkyne compounds, i.e. phenylacetylene (Table 6, reaction 3) and 3,5-bis(hydroxymethyl)-1propargyloxybenzene (PBM) (Table 6, reaction 4). These compounds have been used in recent 'click' research of the authors. ${ }^{[39]}$ In all reactions (table 2), DMF was used as solvent and

Regardless of the copper concentration used, filtration of the postreaction mixtures resulting from the cycloaddition of azido-PEO and phenylacetylene (Table 2, entries 1 and 2) gave similar results with regard to catalyst (52\%) and product (93\%) retention. Product rejections were even slightly higher (96\%) when azido-PEO was reacted with PBM. In this case, a significantly higher catalyst rejection was obtained when a higher catalyst concentration was used in the reaction (Table 2, entriy 3). In contrast to reaction 4 and rather unexpectedly, the permeate flux was higher at a lower substrate/catalyst loading in the filtration of post-reaction mixture 3. Overall, the catalyst rejections clearly show that one single filtration is not sufficient to remove the copper until below the threshold concentration limit.

Based on its elevated product rejection and superior permeability, reaction 3b (Table 2, entry 2) was selected for further filtration experiments with membrane M3, aiming at a further reduction of the copper content in the retained product.

\section{Product purification via diafiltration}

Since full purification of the final 'click' polymer formed in reaction 3b (Table 6) could not be achieved in one single filtration with membrane M3, a post-reaction diafiltration was carried out in which a progressive 'washing out' of the lower molecular weight compounds, i.e. the copper catalyst, was anticipated, thus achieving a higher purity of the retained 'click' product. This was realized by adding fresh solvent (DMF) to the feed reservoir after permeation of approximately $50 \%$ of the initial post-reaction mixture. This discontinuous dilution of the retentate with DMF was repeated four times, in such a way that the feed volume of each new filtration was always equal to the initial feed volume $(50 \mathrm{ml})$. In all five filtrations, the same membrane coupon was used. The diafiltration process is schematically shown in Figure 2 and the fluxes and separation efficiencies of the five successive membrane filtrations are summarized in Table 3. 


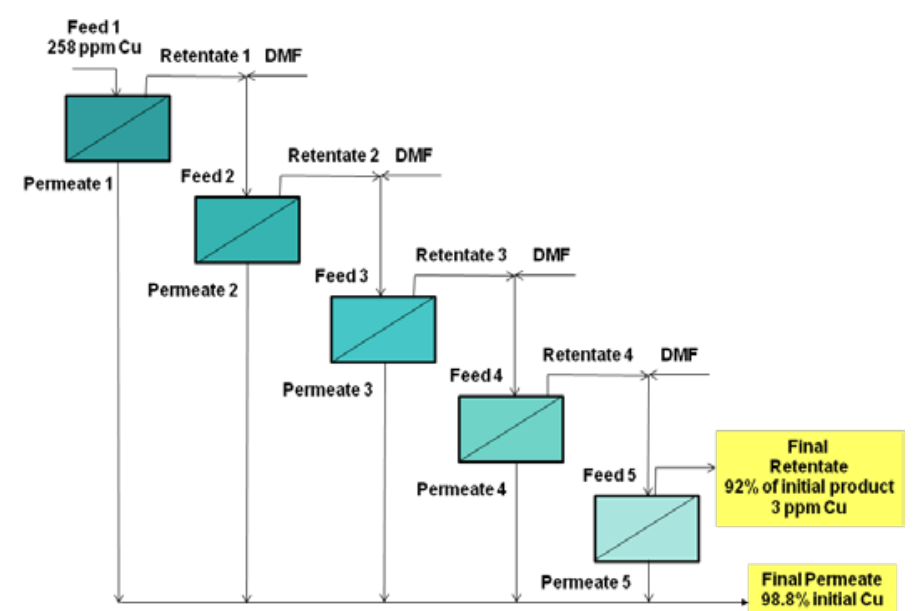

Figure 2. Schematic overview of the diafiltration process. Reaction 3b. Filtration conditions: membrane M3,10 bar, room temperature.

Approximately $50 \mathrm{ml}$ of reaction mixture (product yield: 92\%) was poured into the filtration cell. This solution (Feed 1), with an initial copper concentration of $258 \mathrm{ppm}$ as determined by UV-VIS spectroscopy, was then filtrated at 10 bar till approximately half of the initial feed had permeated through the membrane. The filtrate (Permeate 1) was collected and analyzed for copper content via UVVis spectroscopy and the presence of polymer both gravimetrically and via ${ }^{1} \mathrm{H}$ NMR spectroscopy. Subsequently, the remaining solution (Retentate 1) was depressurized and diluted with fresh DMF back to its initial volume. This new mixture (Feed 2) was filtrated again over the same membrane. After the second filtration of approximately half of Feed 2, only $3.67 \mathrm{mg}$ of the initial 14.17 mg of copper remained at the retentate side (Retentate 2), constituting a 75\% reduction (Figure 3 ). With product selectivities up to $96 \%$, only $4 \%$ of the polymer was lost through the membrane (Permeate 2), confirming the rejections found in the screening test (Table 2, entry 2). In the three subsequent filtrations, no further product loss was observed (Table 3). After five filtrations and four dilutions, $92 \%$ of the total polymer present in Feed 1 was thus recovered at the retentate side of the membrane (Figure 2). A gradual and almost complete removal of the copper could be detected (Table 3), until $3 \mathrm{ppm}$ in the final retentate, meaning that $98.8 \%$ of the initial catalyst had been removed (Figure 3). This final copper concentration of the product mixture is well below the copper residue limit (15 ppm) prescribed by the European Medicines Agency, ${ }^{[9]}$ thus demonstrating the feasibility of the proposed diafiltration process. As anticipated from the screening tests, no copper fouling was observed on the membrane surface.

Table 3. Permeabilities, product rejections and retentate copper concentrations of the five successive filtrations of the diafiltration process. Reaction 3b. Filtration conditions: membrane M3,10 bar, room temperature.

\begin{tabular}{cccc}
\hline Sample & $\begin{array}{c}\text { Permeability } \\
\left(\mathbf{l . m}^{-\mathbf{2}} \mathbf{h}^{\mathbf{- 1}} \cdot \mathbf{b a r}^{-\mathbf{1}} \mathbf{)}\right.\end{array}$ & $\begin{array}{c}\text { Rejection polymer } \\
\text { product (\%) }\end{array}$ & $\begin{array}{c}\text { Copper concentration in } \\
\text { retentate (ppm) }\end{array}$ \\
\hline Feed & - & - & 258 \\
Filtration 1 & 1.1 & 96 & 118 \\
Filtration 2 & 1.9 & 96 & 69 \\
Filtration 3 & 1.9 & 100 & 28 \\
Filtration 4 & 1.9 & 100 & 13 \\
Filtration 5 & 2.4 & 100 & 3 \\
\hline
\end{tabular}

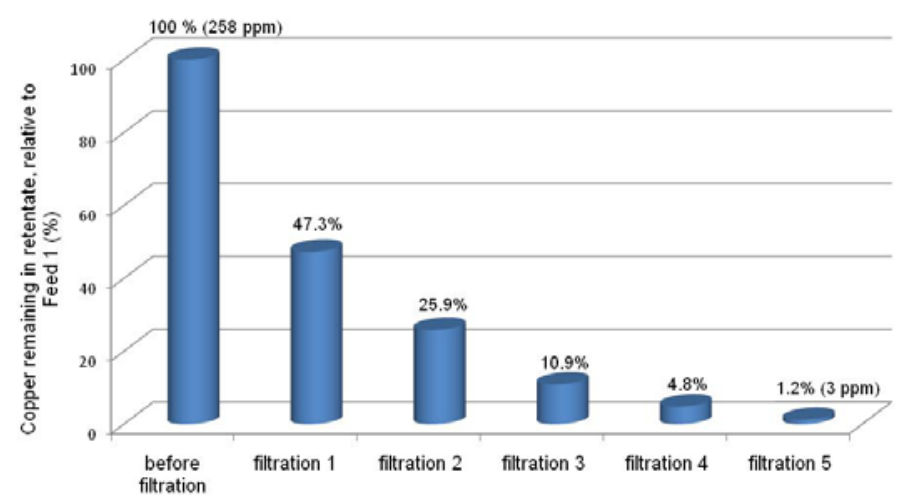

Figure 3. Evolution of the copper concentration upon diafiltration of postreaction mixture 3 (Table 6 ) with membrane M3 (10 bar, room temperature).

In parallel with the progressive copper removal, a significant DMF permeability increase was observed, from $1.1 \mathrm{~lm}^{-2} \cdot \mathrm{h}^{-1} \cdot \mathrm{bar}^{-1}$ in the first filtration to $2.4 \mathrm{l} \cdot \mathrm{m}^{-2} \cdot \mathrm{h}^{-1} \cdot \mathrm{bar}^{-1}$ in the last filtration (Table 3). This rather unexpected flux increase cannot be explained by a gradual degradation of the membrane in the solvent medium since the polymer rejection even increased during the diafiltration process. Possibly, a swelling of the membrane took place during the extended exposure to DMF, also overnight under non-pressurized conditions.

The ratio between the mass of 'click' product to the mass of copper in the initial reaction mixture (feed 1) was found to be 68. After the 5-step diafiltration experiments, the same ratio in retentate 5 was 5806), thus increased to 85 fold (compared to the feed 1 value. Thanks to its favourable product/copper selectivity, high flux and good DMF stability throughout the entire diafiltration process, the developed crosslinked PI membrane allows to separate 'clicked' polymer compounds from the copper catalyst, thus offering an efficient tool for product purification.

\section{Conclusion}

SRNF was presented as a powerful tool to separate 'click' reaction mixtures, aiming at an efficient purification of the formed polymeric products. The developed crosslinked PI membranes permitted permeation of the ligand-stabilized copper (I) catalyst, while the products were almost fully retained. This is the first SRNF report where the catalyst is the permeating species and the product is retained. After an initial screening, the most promising membrane was used in a diafiltration experiment involving five consecutive filtrations and intermediate dilutions with DMF. This allowed to remove almost $99 \%$ of the initial copper, resulting in a metal content in the product stream of no more than $3 \mathrm{ppm}$. Thanks to their favourable product/copper selectivity, elevated flux and excellent DMF stability, the developed membranes offer a robust alternative for the current, generally limited technologies to purify 'click' products. The membranes with tailor-made MWCO offer a powerful and general solution for post-reaction product work-up, thus responding to one of the most important issues in the burgeoning field of 'click' chemistry.

\section{Experimental Section}

\section{Synthesis of 'click’ reagents}

3,5-bis(hydroxymethyl)-1-propargyloxybenzene $\quad$ (PBM), ${ }^{[45]} \mathrm{N}-2$ (azidoethyl)phthalimide (PHT- $\left.\mathrm{N}_{3}\right),{ }^{[46]}$ and azido-poly(ethylene oxide) (azido-PEO) $(1125 \mathrm{~g} / \mathrm{mol} \text { and } 2000 \mathrm{~g} / \mathrm{mol})^{[47]}$ were synthesized as reported in literature. The polyurethane PU-PBM-50 (8100 g/mol) was synthesized from 1 equivalent of PBM and 1 
equivalent of hexamethylene diisocyanate (HDI) according to Fournier et al. ${ }^{[46]}$

Copper(I) bromide (CuBr, 99.99\%), $\quad N, N, N^{\prime}, N^{\prime}, N^{\prime \prime}$ pentamethylethylenetriamine (PMDETA, 99+\%), phenylacetylene (98\%), tetrahydrofuran (THF, 99+\%) and dimethylformamide (DMF, 99+\%) were used as received. All reagents were obtained from commercial suppliers and used without further purification.

\section{Preparation of SRNF membranes}

Integrally skinned asymmetric SRNF membranes were prepared from Lenzing P84 ${ }^{\circledR}$ polyimide (PI) dope solutions via the phaseinversion technique. The polymer was purchased from Evonik (Germany) as a $25 \mathrm{wt} \%$ solution in N-methylpyrrolidinone (NMP, 99\%), which was further diluted in THF:NMP mixtures with a 1:5 or 1:3 solvent weight ratio. The three casting solutions with compositions indicated in Table 5 were cast as $250 \mu \mathrm{m}$ thick film on a non-woven support (Novatex 2471, Freudenberg, Germany) using an automatic film applicator (Braive Instruments, Belgium). The nascent films were then shortly exposed to ambient air for $60 \mathrm{~s}$ to allow solvent evaporation from the surface, and subsequently immersed in a de-ionized water bath at room temperature, where solidification of the membrane structure took place. ${ }^{[28,41]}$

Table 5. Composition of the casting solutions of the PI membranes.

\begin{tabular}{ccccc}
\hline Membrane & $\begin{array}{c}\text { P84 } \\
\text { (w\%) }\end{array}$ & $\begin{array}{c}\text { NMP } \\
\text { (w\%) }\end{array}$ & $\begin{array}{c}\text { THF } \\
\text { (w\%) }\end{array}$ & $\begin{array}{c}\text { THF:NMP } \\
\text { solvent ratio }\end{array}$ \\
\hline M1 & 21.5 & 65.4 & 13.1 & $1: 5$ \\
M2 & 19.0 & 66.7 & 13.3 & $1: 5$ \\
M3 & 19.0 & 60.7 & 20.3 & $1: 3$ \\
\hline
\end{tabular}

Chemical crosslinking was achieved by immersing the PI membranes for $24 \mathrm{~h}$ in a $10 \mathrm{wt} \%$ p-xylylenediamine (99\%) solution in methanol (99\%). ${ }^{[39]}$ After crosslinking, the membranes were extensively rinsed with methanol to wash out the crosslinker, and then post-treated via solvent exchange, involving immersion in 2propanol (IPA, 99\%) for 3h, and subsequently for 3 days in a toluene/2-methyl-4-pentanone/mineral oil (P3, Pfeiffer) solution with a $40 / 40 / 20$ volume ratio. ${ }^{[48]}$ Finally, the membranes were dried for $1 \mathrm{~h}$ at $60^{\circ} \mathrm{C}$, and stored until use.

\section{‘Click’ reactions}

'Click' coupling reactions were carried out for $14 \mathrm{~h}$ at room temperature using DMF and occasionally THF as a solvent. In all cases 1 equivalent of polymeric compound and 2 equivalents of organic compound were mixed together with the catalyst based on $\mathrm{CuBr}$ and the ligand PMDETA. The structure and molecular weight of the reacting compounds, and the equivalents of copper salt and ligand are summarized in Table 6 .

Table 6. Reaction conditions of the copper (I) catalyzed 1,3-dipolar cycloadditions in DMF.

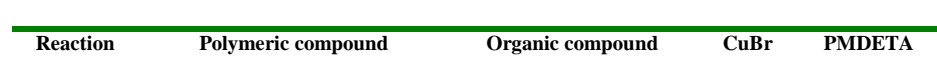

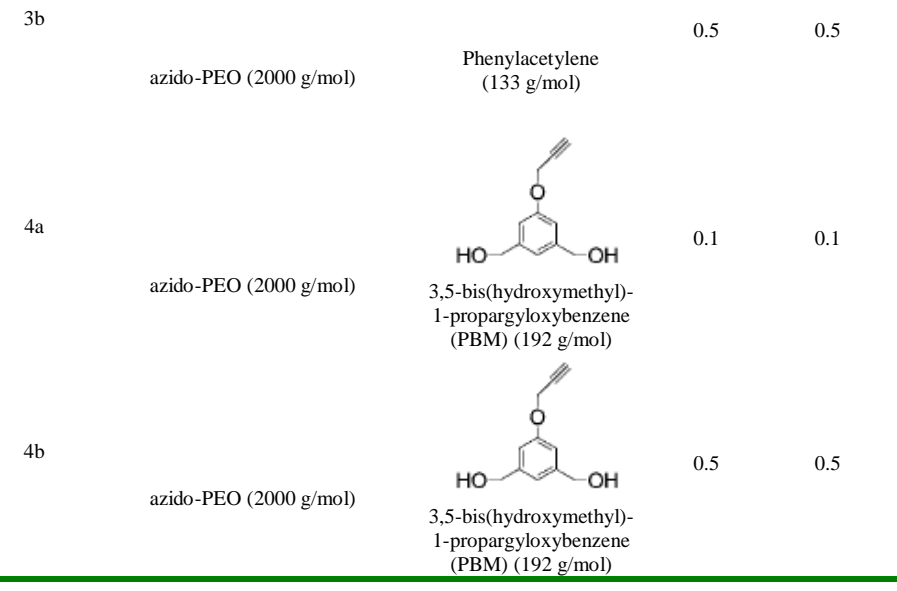

\section{Filtration experiments}

Room temperature filtration experiments were carried out on circular membrane coupons of $12.6 \mathrm{~cm}^{2}$ using a stainless steel deadend filtration cell. Approximately $50 \mathrm{ml}$ of post-reaction mixture was poured into the cell, magnetically stirred at $500 \mathrm{rpm}$, and pressurized to 10 bar with nitrogen gas. In the screening tests, permeate samples were only taken after initial non-equilibrium conditions during which the permeates were discarded. Permeabilities $\left(\mathrm{l}^{-2} \mathrm{~m}^{-1} \cdot \mathrm{h}^{-1} \cdot \mathrm{bar}^{-1}\right)$ were determined gravimetrically.

In the diafiltration experiment, the permeate was collected immediately after pressurization until approximately half of the initial feed volume $(50 \mathrm{ml})$ had permeated. After filtration, the retentate was depressurized and approximately $25 \mathrm{ml}$ of fresh DMF was added after which the new feed was filtrated again. These discontinuous filtration-dilution cycles with a constant feed volume at the start of each filtration, were repeated until achieving the desired degree of purification.

Product and catalyst rejections (\%) were calculated as $\left(1-\mathrm{C}_{\mathrm{p}} / \mathrm{C}_{\mathrm{f}}\right) \mathrm{x}$ 100 , where $C_{f}$ and $C_{p}$ refer to the solute concentration of the initial feed and of the permeate, respectively. Catalyst concentrations were analyzed on a Perkin-Elmer Lamda 12 UV-Vis spectrophotometer at $697 \mathrm{~nm}$. The concentration of 'Click' products was gravimetrically determined after evaporation of the solvent from the difference of weight between the empty sample container and the weight after evaporating the solvent. If this weight difference was lower than $0.001 \mathrm{~g}$ it was considered that no product was present in the sample. Since the maximum amount of copper catalyst at the beginning of the reaction was $32 \mathrm{mg}$ and considering that most of it had been removed, it can be assumed that the weight difference is only due to the presence of the 'click' product.

${ }^{1} \mathrm{H}$ NMR spectroscopy was used to identify the presence of the 'click' polymer in the samples. ${ }^{1} \mathrm{H}$ NMR spectra were recorded in $\mathrm{CDCl}_{3}$ and DMSO- $d_{6}$ at room temperature on a Bruker Avance 300 at $300 \mathrm{MHz}$. 


\section{Acknowledgements}

This research was done in the framework of an I.A.P.-P.A.I. grant (IAP 6/27) sponsored by the Belgian Federal Government, and of a G.O.A. grant from K.U.Leuven. A. CanoOdena acknowledges the financial support from the Departament d'Universitats, Recerca $i$ Societat de la Informació (DURSI) from Generalitat de Catalunya for a postdoctoral scholarship (ref 2006 BP-A 10012). I.F.J. Vankelecom and A. Cano-Odena acknowledge the Long term Methusalem funding by the Flemish Government. B. De Meyer and A. Ramaswamy from Ghent University are also acknowledged for their contribution in sample analysis.

[1] H. C. Kolb, M. G. Finn and K. B. Sharpless, Angew. Chem., Int. Ed. 2001, 40, 20042021.

[2] G. C. Tron, T. Pirali, R. A. Billington, P. L. Canonico, G. Sorba and A. A. Genazzani, Med. Res. Rev. 2008, 28, 278-308.

[3] B. Le Droumaguet and K. Velonia, Macromol. Rapid Commun. 2008, 29, 10731089.

[4] W. H. Binder and R. Sachsenhofer, Macromol. Rapid Commun. 2008, 29, 952-981.

[5] J. E. Moses and A. D. Moorhouse, Chem. Soc. Rev. 2007, 36, 1249-1262.

[6] D. Fournier, R. Hoogenboom and U. S. Schubert, Chem. Soc. Rev. 2007, 36, 13691380 .

[7] H. Nandivada, X. W. Jiang and J. Lahann, Adv. Mater. 2007 19, 2197-2208.

[8] Y. Shen, H. Tang and S. Ding, Prog. Polym. Sci. 2004, 29, 1053-1078.

[9] The European Agency for the Evaluation of Medicinal Products. http://www.emea.europa.eu/pdfs/human/swp/44600en.pdf, 2007.

[10] S. T. Laughlin, J. M. Baskin, S. L. Amacher and C. R. Bertozzi, Science 2008, 320 664-667.

[11] X. H. Ning, J. Guo, M. A. Wolfert and G. J. Boons, Angewandte ChemieInternational Edition 2008, 47, 2253-2255.

[12] J. F. Lutz, Angewandte Chemie-International Edition 2008, 47, 2182-2184.

[13] C. R. Becer, R. Hoogenboom and U. S. Schubert, Angewandte ChemieInternational Edition 2009, 48, 4900-4908.

[14] H. Durmaz, A. Dag, O. Altintas, T. Erdogan, G. Hizal and U. Tunca, Macromolecules 2007, 40, 191-198.

[15] K. L. Killops, L. M. Campos and C. J. Hawker, J. Am. Chem. Soc. 2008, 130 5062-+.

[16] A. J. Inglis, S. Sinnwell, M. H. Stenzel and C. Barner-Kowollik, Angewandte Chemie-International Edition 2009, 48, 2411-2414.

[17] D. J. Cole-Hamilton and R. P. Tooze, Catalyst Separation, Recovery and Recycling - Chemistry and Process Design, Springer, The Netherlands, 2006, p.

[18] K. Matyjaszewski, T. Pintauer and S. Gaynor, Macromolecules 2000, 33, 14761478.

[19] L. Bonami, W. Van Camp, D. Van Rijckegem and F. Du Prez, Macromol. Rapid Commun. 2009, 30, 34-38.

[20] A. J. Carmichael, D. M. Haddleton, S. A. F. Bon and K. R. Seddon, Chem Commun. 2000, 1237-1238.

[21] T. Biedron and P. Kubisa, Macromol. Rapid Commun. 2001, 22, 1237-1242.

[22] J. Xia, T. Johnson, S. G. Gaynor, K. Matyjaszewski and J. DeSimone, Macromolecules 1999, 32, 4802-4805.

[23] G. Kickelbick, H.-J. Paik and K. Matyjaszewski, Macromolecules 1999, 32, $2941-$ 2947.

[24] D. Fournier, S. Pascual, V. Montembault and L. Fontaine, J. Polym. Sci., Part A: Polym. Chem. 2006, 44, 5316-5328.
[25] S. Ding, J. Yang, M. Radosz and Y. Shen, J. Polym. Sci., Part A: Polym. Chem. 2003, 42, 22-30.

[26] Y. Shen, S. Zhu and R. Pelton, Macromolecules 2001, 34, 3182-3185.

[27] D. E. Bergbreiter, Chem. Rev. 2002, 102, 3345-3384.

[28] P. Vandezande, L. E. M. Gevers and I. F. J. Vankelecom, Chem. Soc. Rev. 2008, 2 , 365-405.

[29] C. Müller, M. G. Nijkamp and D. Vogt, Eur. J. Inorg. Chem. 2005, 2005, 40114021.

[30] A. Livingston, L. Peeva, S. Han, D. Nair, S. S. Luthra, L. S. White and L. M. Freitas dos Santos, Ann. N.Y. Acad. Sci. 2003, 984, 123-141.

[31] H. P. Dijkstra, G. P. M. Van Klink and G. Van Koten, Acc. Chem. Res. 2002, 35, 798-810.

[32] I. F. J. Vankelecom, Chem. Rev. 2002, 102, 3779-3810.

[33] A. Datta, K. Ebert and H. Plenio, Organometallics 2003, 22, 4685-4691.

[34] S. Aerts, A. Buekenhoudt, H. Weyten, L. E. M. Gevers, I. F. J. Vankelecom and P. A. Jacobs, J. Membr. Sci. 2006, 280, 245-252.

[35] A. Keraani, T. Renouard, C. Fischmeister, C. Bruneau and M. Rabiller-Baudry, ChemSusChem 2008, 1, 927-933.

[36] G. Baumgarten in Vol. Aachen (Germany), March 27-29, 2007.

[37] C. J. Pink, H. Wong, F. C. Ferreira and A. G. Livingston, Org. Proc. Res. Dev. 2008, 12, 589-599.

[38] M. Janssen, C. Müller and D. Vogt, Adv. Synth. Catal. 2009, 351, 313-318.

[39] K. Vanherck, P. Vandezande, S. O. Aldea and I. F. J. Vankelecom, J. Membr. Sci. 2008, 320, 468-476.

[40] Y. H. See Toh, F. W. Lim and A. G. Livingston, J. Membr. Sci. 2007, 301, 3-10.

[41] I. F. J. Vankelecom, K. De Smet, L. E. M. Gevers and P. A. Jacobs in Vol. Eds. A. I. Schäfer, A. G. Fane and T. D. Waite), Elsevier, Oxford, 2005, pp. 33-65.

[42] G. Moroi, React. Funct. Polym. 2008, 68, 268-283.

[43] P. Vandezande, X. Li, L. E. M. Gevers and I. F. J. Vankelecom, J. Membr. Sci. 2009, 330, 307-318.

[44] Y. H. See-Toh, F. C. Ferreira and A. G. Livingston, J. Membr. Sci. 2007, 299, 236250

[45] M. J. Joralemon, R. K. O'Reilly, J. B. Matson, A. K. Nugent, C. J. Hawker and K. L. Wooley, Macromolecules 2005, 38, 5436-5443.

[46] D. Fournier and F. Du Prez, Macromolecules 2008, 41, 4622-4630.

[47] L. Billiet, D. Fournier and F. E. Du Prez, J. Polym. Sci., Part A: Polym. Chem 2008, 46, 6552-6564.

[48] P. Vandezande, L. E. M. Gevers, J. S. Paul, I. F. J. Vankelecom and P. A. Jacobs, J. Membr. Sci. 2005, 250, 305-310.

Received: ((will be filled in by the editorial staff)) Revised: ((will be filled in by the editorial staff)) Published online: ((will be filled in by the editorial staff)) 
Entry for the Table of Contents (Please choose one layout only)

Layout 1:

\section{Catch Phrase}

A. Cano-Odena,

P. Vandezande ${ }^{[a],[1]}$

D. Fournier, ${ }^{[b],\left[{ }^{\prime}\right]}$ W. Van Camp, ${ }^{[b]}$

F. E. Du Prez, ${ }^{[b]}$

F.J. Vankelecom ${ }^{[\mathrm{a}],[*]}$

I.

Page - Page

Solvent resistant nanofiltration for product purification and catalyst recovery in click chemistry reactions

Layout 2:

Catch Phrase

Author(s), Corresponding

Author(s)* Page - Page

\section{Title Text}

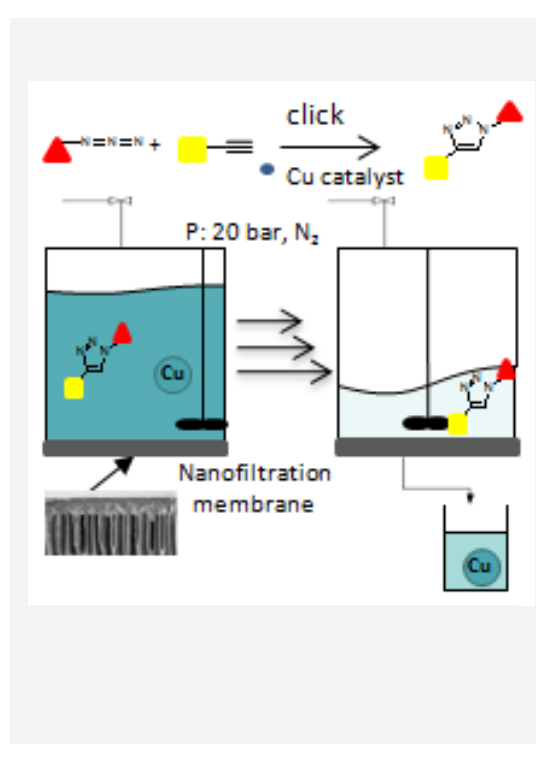

A diafiltration experiment using solvent resistant nanofiltration membranes enabled the almost complete copper catalyst removal and, therefore, the purification of click chemistry products . For first time, the homogeneous catalyst pemeates through a nanofiltration membrane while the purified product is retained.
((The TOC Graphic should not exceed the size of this area))

Text for Table of Contents, max. 450 characters.
Text for Table of Contents, continued. 